\title{
Relations fournisseurs-grande distribution dans les filières agroalimentaires. Une analyse de la trajectoire d'une démarche « filière qualité »
}

Relationships suppliers-retailer in food chains: the processual analysis of the path of a retailer's "food chain brand" initiative

\section{Virginie Baritaux et Marie Houdart}

\section{(2) OpenEdition}

\section{Journals}

Édition électronique

URL : http://journals.openedition.org/economierurale/4598

DOI : 10.4000/economierurale.4598

ISSN : 2105-2581

Éditeur

Société Française d'Économie Rurale (SFER)

Édition imprimée

Date de publication : 15 mars 2015

Pagination : 15-30

ISSN : 0013-0559

Référence électronique

Virginie Baritaux et Marie Houdart, « Relations fournisseurs-grande distribution dans les filières agroalimentaires. Une analyse de la trajectoire d'une démarche « filière qualité »», Économie rurale [En ligne], 346 | Mars-avril 2015, mis en ligne le 15 mars 2017, consulté le 07 mai 2019. URL : http:// journals.openedition.org/economierurale/4598; DOI : 10.4000/economierurale.4598 


\title{
Relations fournisseurs-grande distribution dans les filières agroalimentaires Une analyse de la trajectoire d'une démarche "filière qualité "
}

\author{
Virginie BARITAUX • Clermont Université, VetAgro Sup, UMR Métafort, Lempdes \\ virginie.baritaux@vetagro-sup.fr \\ Marie HOUDART • Irstea, UMR Metafort, Aubière \\ marie.houdart@irstea.fr
}

L'objectif des auteures est d'analyser la nature des relations fournisseurs-distributeurs dans le cadre d'une démarche de type " filière qualité ». Elles étudient le cas d'une filière impliquant une laiterie indépendante et des éleveurs, pour la valorisation de fromages d'Appellation d'origine protégée. Elles abordent ces relations en s'appuyant sur le cadre d'analyse interactionniste du groupe IMP. Un cadre d'analyse processuelle est mobilisé pour établir la trajectoire de cette démarche. Les auteures montrent notamment le rôle des différents acteurs dans la construction de la relation.

MOTS-CLÉS : grande distribution, analyse processuelle, analyse interactionniste, marque filière, rapports de force, filières agroalimentaires

\section{Relationships suppliers-retailer in food chains: the processual analysis of the path of a retailer's "food chain brand" initiative}

Authors aim at analyzing suppliers-retailer relationships within the framework of supermarkets chains' branding initiatives. They study the case of a French supermarkets chain's own "quality food chain" for two blue cheeses with a Protected Designation of Origin (PDO). This branding strategy is based on a partnership with an independent dairy and milk producers for producing differentiated PDO cheeses. They use the interactionist analysis framework developed by the IMP group. Authors carry a processual analysis framework to conduct a dynamic analysis and characterize the path of this initiative. They show that each category of stakeholders influences the path at some stage. (JEL: L66, Q130, L81).

KEYWORDS: supermarket chains, processual analysis, interactionist analysis, supermarkets own brands, power relationships, food chains

\footnotetext{
a question de l'équilibre des rela-tions entre la grande distribution et les acteurs de l'amont des systèmes agroalimentaires se pose depuis plusieurs décennies (Pouzenc et Guibert, 2010). Sa forte concentration et son internationalisation donnent aux distributeurs un poids croissant dans les systèmes agroalimentaires au niveau global comme au niveau local. Cette évolution a des conséquences non seulement sur le partage de la valeur, mais également en termes d'organisation des
}

acteurs et de définition des produits et des normes de production (Burch et Lawrence, 2007). Pourtant, depuis les années 1990, la nature des relations entre la grande distribution et ses fournisseurs a connu une inflexion (Lepers, 2003). À cette période, deux tendances fortes s'imposent aux distributeurs (Daumas, 2006). D'une part, ils font face à une pression croissante de la part de leurs concurrents directs, mais également des grands groupes de l'industrie agroalimentaire, de plus en plus concentrée 
elle aussi. D'autre part, à la suite des différentes crises sanitaires, les consommateurs expriment des attentes fortes en termes de qualité des produits (éthique, sanitaire, typicité). Les distributeurs mettent alors en place des stratégies de différenciation qui reposent sur le développement d'une offre de produits sous marques propres répondant à des cahiers des charges spécifiques, les Marques de distributeurs (MDD) et les " marques de filière ». Alors que les premières reposent sur une contractualisation bilatérale entre une PME et une enseigne de distribution (Messeghem, 2005), les marques de filière reposent sur des démarches de certification tripartites qui associent un industriel, des producteurs agricoles et le distributeur par le biais de systèmes verticaux de contractualisation pilotés par ce dernier (de Fontguyon et al., 2003 ; Mazé, 2002 ; Réviron et Chappuis, 2005). Ces démarches présentées comme coopératives et fondées sur des partenariats avec les acteurs de l'amont des filières ont été un outil pour les distributeurs pour renforcer leur légitimité et communiquer sur leur engagement sociétal (Beylier et al., 2012 ; Messeghem, 2005). Néanmoins, le déséquilibre des rapports de force pousse à s'interroger sur la réelle nature de ces relations. Pour certains, le développement de ces démarches traduit la montée en puissance des distributeurs dans les systèmes agroalimentaires (Burch et Lawrence, 2005 ; Filser et Paché, 2008) et ces stratégies représentent un moyen de renforcer leur pouvoir sur les autres acteurs des filières (Allain et Chambolle, 2003 ; Berges-Sennou et Caprice, 2003 ; Filser, des Garets et Paché, 2001 ; Fonte, 2008).

Cependant, d'autres travaux relativisent cette vision. Antéblian-Lambrey (1998) insiste sur le développement des approches «plus relationnelles que transactionnelles » dans les échanges entre la grande distribution et ses fournisseurs. Elle souligne le fait que, malgré leur position dominante dans les filières, les distributeurs doivent établir des relations de long terme avec leurs fournisseurs, relations de coopération fondées sur un engagement des deux parties (via des investissements spécifiques notamment). Messeghem (2005), Sabri et Messeghem (2012) ou encore Cox et Chicksand (2007) montrent ainsi que, malgré une logique globale d'affrontement, les MDD peuvent laisser la place à des coopérations entre les PME agroalimentaires et les distributeurs, bénéfiques aux deux parties. Mazé (2002) met également en évidence l'intérêt, pour les éleveurs, de s'engager dans un dispositif de certification de type "marque de filière ». Néanmoins, elle se focalise essentiellement sur les dispositifs contractuels et sur l'impact qu'ils ont sur le pouvoir de négociation dont disposent les différents acteurs de la filière, et elle n'analyse pas réellement la nature de la relation qui s'établit entre les parties prenantes.

Notre objectif est de caractériser et d'analyser la nature des relations entre un distributeur et ses fournisseurs dans le cadre d'une démarche de type « filière qualité ». Comment s'articulent les stratégies des différentes parties prenantes ? Quel rôle jouent-elles dans la construction et la vie de la démarche ?

Il s'agit plus précisément de comprendre comment se construit et se déroule une relation de coopération tripartite entre une enseigne de la grande distribution, une PME agroalimentaire et des producteurs agricoles, dans le cadre d'une démarche de type partenariat vertical pour la mise en place d'une « filière qualité ». Pour cela, nous nous appuyons sur le cas d'une filière qualité sur deux fromages $\mathrm{AOP}$ impliquant un distributeur, une laiterie et des éleveurs laitiers. Nous abordons les relations entre le distributeur et ses fournisseurs en nous appuyant sur le cadre d'analyse interactionniste développé par les auteurs du groupe Industrial Marketing 
and Purchasing (IMP) (Nogatchewsky et Donada, 2005). L'interdépendance entre les organisations est à l'origine de relations de long terme au cours desquelles se mettent en place des échanges sociaux et d'information, financiers et de biens ou de services (Leek, Turnbull et Naudé, 2001). Les relations inter-organisationnelles sont ainsi conçues comme des processus d'interaction dont l'analyse nécessite de prendre en compte leur dimension temporelle (Ford et Håkansson, 2006). Nous mobilisons donc le cadre d'analyse développé par Mendez et al. (Mendez, 2010) qui permet d'analyser et de comprendre la trajectoire de processus complexes.

La première partie de l'article revient sur l'intérêt des approches interactionnistes pour l'analyse des relations entre la grande distribution et ses fournisseurs et présente le modèle d'interaction du groupe IMP. Dans un second temps, le cadre d'analyse processuelle ainsi que la méthodologie sont présentés. La trajectoire de la relation fondant la filière qualité est analysée dans une troisième partie. La quatrième partie discute ces résultats.

\section{Analyse des relations producteurs-distributeurs Une approche interactionniste}

\section{L'intérêt de l'approche interactionniste pour l'analyse des relations fournisseurs- distributeurs}

La littérature abondante sur les relations clients-fournisseurs peut se répartir dans deux grands courants, celui du canal marketing $^{1}$ et le courant interactionniste (Nogatchewsky et Donada, 2005). Notre réflexion s'inscrit dans ce second courant, porté en grande partie par des chercheurs européens (Cova et Salle, 1992)

1. Le courant du canal marketing regroupe des travaux portant sur les fonctions économiques au sein du canal, les comportements des acteurs et les modes de gouvernance des échanges. regroupés notamment au sein du groupe $\mathrm{IMP}^{2}$. Ces recherches visent à comprendre la dynamique des interactions et les relations d'interdépendance qui s'établissent entre les partenaires impliqués dans une relation commerciale. Les relations interorganisationnelles y sont conçues comme des processus d'interaction. Ces approches permettent de prendre en compte la dimension « relationnelle » des échanges en considérant l'interdépendance des organisations et en se plaçant dans une perspective de long terme (Cova et Salle, 1992).

Comme le souligne Antéblian-Lambrey (1998), appliquées au cas des relations producteurs-distributeurs, ces approches offrent un intérêt dans la mesure où elles permettent d'élargir une analyse essentiellement basée sur les notions de pouvoir et de conflit développées notamment par les approches comportementales des canaux de distribution (Hunt, Ray et Van Wood, 1985 ; Stern, 1969) ${ }^{3}$ et de prendre en compte des formes d'échange plus relationnelles, basées sur de la coopération, organisées dans le temps, et qui pourraient s'établir dans les canaux de distribution. En effet, les approches développées par les interactionnistes reposent sur deux grands principes fondateurs (Skytte, 2010). Selon le premier principe, il convient de se focaliser sur des relations et non sur des transactions « discrètes » (Dwyer, Schurr et Oh, 1987). On privilégie alors des approches sur le long terme et on prend en compte le fait que les interactions présentes se placent dans une histoire et sont liées aux interactions passées et futures (Ford et Håkansson, 2006). Ce type d'approche permet donc plus facilement d'envisager des relations de coopération sur du long

2. Pour plus d'information voir le site Internet www.impgroup.org.

3. Pour une présentation détaillée des différents courants de pensée dans la littérature sur les canaux de distribution, voir notamment Filser (2000) ou Wilkinson (2001). 
terme entre producteurs et distributeurs. Le second principe consiste à considérer que l'ensemble des organisations en interaction sont actives, le fournisseur comme le client. Les adaptations liées aux interactions ne sont alors pas forcément univoques et sous l'influence d'une seule organisation : elles dépendent des rapports de force entre les organisations qui peuvent être aussi bien en faveur du fournisseur que du client (Cova et Salle, 1992). Ce principe amène à aborder les relations entre fournisseurs et distributeurs comme des relations dont l'orientation et l'organisation ne sont pas uniquement contrôlées par le distributeur (Antéblian-Lambrey, 1998).

\section{Le modèle d'interaction du groupe IMP}

Les premiers travaux du groupe IMP ont conduit au développement d'un modèle visant à représenter les éléments caractéristiques des relations inter-organisationnelles (Håkansson, 1982). Ce modèle identifie quatre composantes de ces relations.

- La première renvoie aux parties prenantes à l'interaction correspondant non seulement aux organisations mais également aux personnes qui les représentent. Concernant les organisations, des facteurs tels que la position sur le marché (nature de l'activité, taille, etc.), la stratégie, la culture et le fonctionnement ou encore les technologies interviennent. Au sein de ces organisations, les caractéristiques des personnes concernées par la relation (expérience, motivation, nature des relations, etc.) peuvent également influencer le processus d'interaction.

- La relation s'établit dans un environnement général qui influence le processus d'interaction et les parties. Plusieurs aspects sont à prendre en compte : la structure, le dynamisme et le degré d'internationalisation des marchés, la position des parties dans la filière, l'environnement social, culturel, politique et réglementaire.
- L'atmosphère renvoie au «climat» de la relation. Elle influence mais est également le résultat de l'interaction. Elle peut être décrite en termes de pouvoir/ dépendance, de conflit/coopération, de proximité/distance ou d'attentes réciproques entre les parties.

- Enfin, le processus d'interaction doit être décomposé en deux «niveaux », celui de la relation qui se déroule généralement sur une période longue et celui des «épisodes » de court terme qui ponctuent la relation, et au cours desquels quatre types d'échanges s'établissent entre les parties : de produits (ou de services), d'information, financiers et sociaux. Sur le long terme, la relation est façonnée par la forme des échanges (nature, adaptation, négociations, etc.).

\section{La variable temps dans les interactions distributeurs-fournisseurs}

Les approches interactionnistes mettent en avant l'importance de considérer les relations entre organisations non pas comme des séries d'événements prédéterminés mais comme le résultat d'un ensemble d'éléments en interaction qui permettent à la relation de se construire. Le temps apparaît alors comme une dimension centrale pour comprendre et caractériser la nature des relations et des interactions entre les acteurs (Ford et Håkansson, 2006 ; Medlin, 2004). En effet, les interactions sont conçues comme des processus. Un processus peut se définir comme "une série séquentielle d'événements, d'actions et d'activités, individuels et collectifs, qui se déroulent au cours du temps dans un contexte donné $»^{4}$ (Pettigrew, 1997). La compréhension et l'analyse, à un moment donné, d'une relation client-fournisseur nécessitent donc de prendre en compte le

4. "A sequence of individual and collective events, actions, and activities unfolding over time in context." 
présent mais également les événements passés et l'influence possible des événements à venir (Medlin, 2004). Une bonne compréhension des interactions entre les différents acteurs dans le cadre d'une relation de partenariat entre un distributeur et ses fournisseurs nécessite alors de prendre en compte cette dimension processuelle.

\section{Cadre d'analyse, matériel et méthode}

\section{Le cadre de l'analyse processuelle}

Depuis les années 1990, de nombreuses recherches en sciences humaines et sociales ont montré l'intérêt des approches temporelles pour rendre compte des organisations d'acteurs (Ropo, Eriksson et Hunt, 1997). Les analyses en termes de processus ont été largement utilisées dans l'étude de la dynamique des réseaux d'entreprises (Halinen, Medlin et Törnroos, 2012), en particulier par les chercheurs se rattachant au groupe IMP (Bizzi et Langley, 2012).

Ces démarches visent à avoir une vision globale, " holistique », des faits sociaux (Mendez, 2010) en ayant une approche constructiviste du temps (Halinen, Medlin et Törnroos, 2012). Les relations et modes d'organisation entre acteurs sont ainsi le résultat de trajectoires plus ou moins linéaires, qui correspondent à « l'enchaînement de faits dans le temps aboutissant à un résultat, ou un phénomène conçu comme actif et organisé dans le temps » (Mendez, 2010).

Dans cet article, nous nous appuyons sur le cadre d'analyse des processus développé par un collectif de chercheurs français (Mendez, 2010). Ces auteurs, à partir d'une synthèse de différents travaux en sociologie, sciences économiques et de gestion proposent un cadre d'analyse processuelle qui vise à comprendre la combinaison des dynamiques qui fondent les processus. Ces derniers se caractérisent par quatre grandes composantes : les ingrédients, les séquences, les moteurs et les bifurcations. Ces composantes peuvent être reliées aux grandes variables fondant le modèle d'interaction du groupe IMP.

- Les ingrédients sont les éléments du contexte global dans lequel se déroule le processus qui apparaissent pertinents pour comprendre ce dernier. Ils peuvent être conçus comme «des éléments de contrainte qui limitent l'action ou comme des ressources qui la nourrissent » (Mercier et Oiry, 2010). L'identification des ingrédients permet de prendre en compte les caractéristiques de l'environnement général, de l'atmosphère de la relation ou encore des caractéristiques des parties prenantes à la relation qui influencent le processus d'interaction.

- Les séquences sont les « segments temporels » qui s'enchaînent et forment le processus. Elles sont vues comme des configurations particulières qui résultent de l'interaction entre les jeux d'acteurs et les éléments du contexte que sont les ingrédients (Longo, Mendez et Tchobanian, 2010). Les séquences, au cours desquelles des interactions et des échanges de nature aussi bien économique que sociale et/ou informationnelle s'établissent entre les acteurs de la relation, peuvent être associées aux « épisodes » qui ponctuent et constituent la relation de long terme dans le modèle IMP. La trajectoire peut être marquée par un changement d'orientation qui se caractérise par une recomposition importante de la configuration des ingrédients. On parle dans ce cas de bifurcation.

- Enfin, les moteurs sont définis comme des « mécanisme(s) génératif(s) du mouvement des ingrédients et de leurs assemblages au cours du temps » (Pérocheau et Correia, 2010). En s'appuyant sur la typologie proposée par Van de Ven et Poole (1995), Pérocheau et Correia (2010) identifient quatre grands archétypes de moteurs qui peuvent être à 
l'origine d'un mouvement : (i) si on a un moteur programmatique, les séquences suivent un programme qui régule le processus de changement (Brochier et al., 2010) ; (ii) le moteur dit « évolutionniste » résulte de la combinaison de trois types de mécanismes : de variation, de sélection et de « rétention». Dans le cas d'un groupe d'acteurs économiques par exemple, dans un premier temps, des acteurs modifient leurs pratiques. Le mécanisme de sélection conduit ensuite à une conservation ou à un rejet de ces changements par les autres acteurs du système (concurrents, clients...). Enfin, les mécanismes de rétention permettent une conservation et une reproduction des pratiques sélectionnées ; (iii) le moteur « dialectique » permet d'expliquer des mouvements liés à des tensions entre différents ingrédients du processus ; (iv) à l'opposé du précédent, le moteur « téléologique » est présent lorsque c'est " l'existence d'un objectif commun et la tentative d'atteindre cet objectif qui est le principe génératif du mouvement » (Pérocheau et Correia, 2010). Les moteurs permettent de saisir ce qui relève des stratégies des acteurs et influencent la construction et le déroulement de la relation.

Un processus est donc « un ensemble de phénomènes organisés dans le temps qui combine des ingrédients et des moteurs dont le changement éventuel permet d'identifier des séquences. Lorsque ces changements modifient l'orientation même du processus, ils correspondent à des bifurcations » (Brochier et al., 2010). La mise en œuvre de ce cadre d'analyse consiste ainsi, en adoptant une approche historique à identifier la succession des séquences, et les éventuelles bifurcations, qui font la trajectoire, à identifier et analyser la nature des ingrédients et des moteurs, ainsi que la façon dont ils se combinent pour expliquer le processus qui conduit à l'évolution de la relation. Cette approche qui consiste à avoir une approche historique d'un phénomène et à le décomposer en différents éléments pour comprendre le processus aboutissant à cette dynamique permet, comme le recommandent notamment Ford et Håkansson (2006), de prendre en compte les interactions entre les différents épisodes et d'éviter de considérer le processus comme linéaire et prédéterminé.

\section{Matériel et méthode}

\section{Étude de cas}

Nous étudions le cas d'une démarche de certification de type « filière qualité » mise en place par une enseigne de la grande distribution, impliquant une laiterie indépendante et des éleveurs, pour la valorisation de deux fromages sous Appellation d'origine protégée (AOP). Dans le cadre de cette démarche, le distributeur s'engage, outre la garantie de qualité (goût et authenticité des produits, qualité constante, environnement protégé), à soutenir les petits producteurs agricoles et les PME agroalimentaires à travers des partenariats durables et des prix justes.

Créée en 1949, la laiterie est une entreprise familiale qui est l'un des deux acteurs majeurs de la collecte et de la transformation du lait sur le territoire du LivradoisForez, en Auvergne (37\% du lait collecté). Elle transforme environ 40 millions de litres de lait par an, provenant de 195 producteurs. Un tiers de la production est valorisé sous les AOP concernées par la démarche dont environ $25 \%$ sont destinés aux productions au lait cru. En 2012, 19 producteurs étaient engagés dans la démarche.

L'organisation de la filière repose sur une contractualisation tripartite entre le distributeur, la laiterie et ses producteurs. Ces derniers s'engagent à respecter un cahier des charges plus restrictif que ceux des AOP sur trois points. L'alimentation des vaches laitières doit être uniquement 
composée de fourrages secs et d'herbe, éliminant de fait toute alimentation à base de fourrages fermentés ou de maïs. La seconde obligation porte sur l'utilisation exclusive de lait cru pour la fabrication des fromages qui s'accompagne, pour la laiterie, d'une obligation de collecter quotidiennement le lait et de le traiter séparément du lait « standard ». Enfin, les fromages doivent être affinés pendant une période supérieure de plusieurs semaines à la durée minimale définie pour les $\mathrm{AOP}$ « génériques ». En contrepartie du respect de ce cahier des charges, la laiterie s'engage à verser une prime au litre de lait aux éleveurs. Par ailleurs, le distributeur s'engage à acheter un certain volume de fromage à un prix supérieur à celui du produit standard et à le commercialiser, sous marque filière, dans l'ensemble de ses magasins du territoire français disposant d'un rayon à la coupe. Par ailleurs, le distributeur a mis en place un système d'audits et de contrôles des exploitations et de la laiterie pour sélectionner les producteurs et contrôler le respect du cahier des charges.

\section{Collecte et traitement des données}

Outre des données documentaires (cahiers des charges, contrats, articles de journaux...), l'analyse s'appuie sur des données d'enquêtes auprès de différents types d'acteurs. Ce travail s'inscrivant dans un projet de recherche plus large, des entretiens conduits auprès d'acteurs de l'élevage et du territoire ont pu être exploités et ont permis d'avoir une vision large du contexte global dans lequel s'inscrit la démarche.

Pour l'analyse de la trajectoire de la filière étudiée, des entretiens semi-directifs ont été réalisés entre avril et juin 2012. Sept entretiens de cadrage (Bidart et al., 2010), auprès de représentants d'acteurs du territoire et du conseil agricole ayant suivi la trajectoire de cette initiative (consultants et représentants du Parc naturel régional dans lequel se situe la laiterie et les producteurs, de la Chambre régionale d'agriculture, du syndicat interprofessionnel des AOP, de l'association des producteurs de la laiterie, de l'Établissement départemental de l'élevage) ont permis de collecter des informations pour avoir une vision globale du processus. D'autre part, toutes les parties prenantes de la démarche ont été rencontrées, à savoir 18 (sur 19) producteurs engagés dans la démarche, deux représentants de la laiterie et deux représentants de la direction qualité du groupe de distribution. Outre des questions visant à mieux identifier l'activité et les stratégies des différents acteurs, l'évolution générale de la démarche et les événements majeurs ayant marqué sa trajectoire, ces entretiens ont cherché à saisir les motivations individuelles à y participer, ainsi que les caractéristiques techniques de l'activité et des structures de production.

L'analyse qualitative des informations collectées a permis de rendre compte de la trajectoire de la démarche. Cette analyse a été menée en deux phases : analytique et systémique (Bidart et al., 2010). La première a permis d'établir une représentation objective de la chronologie de la construction et du développement de la filière (la matrice temporelle). La démarche est alors représentée comme une succession de dates et d'événements. La phase systémique correspond à l'interprétation et à l'explication de la dynamique de cette chronologie. Elle consiste à mettre en évidence l'articulation entre les séquences, bifurcations, ingrédients et moteurs.

\section{Résultats}

L'analyse de la trajectoire de la démarche conduit à identifier cinq séquences (la figure 1 résume cette trajectoire). Pour chacune de ces séquences, nous avons identifié les ingrédients et les moteurs qui entrent en jeu. 
Figure 1. Composantes de la trajectoire de la démarche

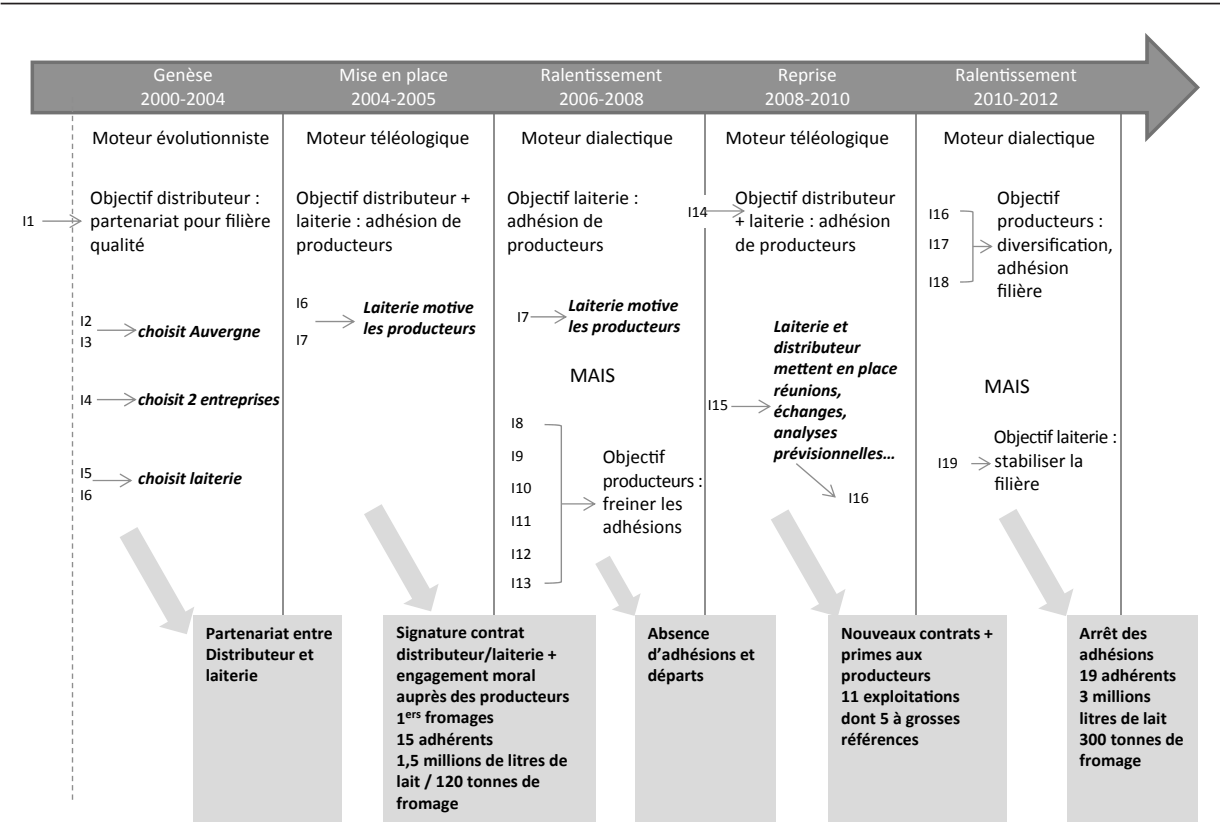

Notes :

I1 - nouvelles attentes des consommateurs et pression concurrentielle croissante pour le distributeur ;

12 - présence d'exploitations valorisant l'herbe et le foin en Auvergne ;

I3 - partenariat préexistant entre le distributeur et une coopérative du Cantal ;

14 - Deux entreprises de transformation répondant aux critères du distributeur : lait tout foin + fromage au lait cru + $\mathrm{AOP}+$ petite entreprise ;

15 - laiterie = PME indépendante + fabrications au lait cru + relations commerciales préexistantes avec le distributeur ;

16 - des exploitations au système fourrager herbe et fourrage sec fournissant la laiterie ;

17 - relations privilégiées entre laiterie et ses producteurs ;

18 - mauvaise presse de la grande distribution auprès des producteurs ;

19 - modification du cahier des charges AOC (moins contraignant) ;

110 - mise en cause des engagements auprès de la CUMA d'ensilage ;

111 - ensilage perçu comme un retour en arrière par certains producteurs ;

112 - année 2007 pluvieuse ;

113 - 2008 : baisse du prix du lait :

114 - nouveau potentiel de vente du distributeur ;

115 - existence d'exploitation " tout foin " performante en Auvergne ;

116 - réseau d'échange sur la technique du séchage en grande ;

117 - contexte régional favorable aux prairies naturelles (AOP, conseil) ;

118 - perspective de suppression des quotas laitiers ;

119 - difficultés du distributeur et nouvelle stratégie.

\section{Séquence 1 : la genèse de la filière} (début des années 2000 - printemps 2004)

On peut percevoir les prémisses de la relation au début des années 2000 lorsque le distributeur engage des démarches pour créer une filière qualité sur deux fromages «bleus ». Dans les années 1990, la grande distribution fait face à une pression concurrentielle croissante et à des nouvelles attentes des consommateurs liées notamment aux différentes crises sanitaires (crise de la listeria, crise de l'ESB) (I1). Dans ce contexte, l'enseigne met en place une stratégie clairement orientée vers le développement d'une offre de produits « socialement responsables ». Cela donne 
lieu à la création de la première filière qualité en 1992. Cette démarche s'étend et évolue dans les années qui suivent. En 2001, des filières qualité existent déjà pour trois fromages fermiers mais l'objectif est de renforcer le dispositif, notamment en créant de nouvelles filières pour disposer, au rayon coupe, d'un plateau de fromages complet. Pour les personnes du groupe engagées dans le développement de ces filières, l'alimentation des animaux à base d'herbe et de foin et le lait cru sont des gages de respect des valeurs défendues par la marque de filière. Dans ce cadre, l'Auvergne est ciblée en raison du nombre important d'exploitations valorisant l'herbe et alimentant les animaux à base d'herbe et de foin (I2). En outre, ce choix est renforcé par l'existence d'un partenariat similaire déjà mis en place avec une coopérative du Cantal pour du Cantal « tout foin » qui a servi de base solide pour définir les fondements de la démarche (cahier des charges notamment) (I3).

Pour répondre à son objectif de développer une filière sur les deux AOP «bleu », le groupe de distribution met en œuvre un dispositif classique de sourcing lui permettant d'identifier les laiteries susceptibles de répondre à sa demande sur le territoire auvergnat. Il aboutit à la sélection de deux entreprises sur la base de différents critères, parmi lesquels la capacité des laiteries à mobiliser des producteurs produisant déjà du lait « tout foin », leur expérience de fabrication de fromages au lait cru et leur ancrage dans le territoire de production des AOP concernées (petite taille, entreprises indépendantes...) (I4). Une fois les partenaires potentiels identifiés, ils sont mis en concurrence via un appel d'offres. Des audits sur les sites de transformation ainsi que des visites dans des exploitations dont les pratiques correspondent déjà au cahier des charges de la filière sont lancés. Au niveau de la direction du distributeur, les dossiers de candidature sont présentés à un jury dont le rôle est d'évaluer la rentabilité économique de ces nouvelles filières. Le processus de sourcing s'achève au printemps 2004 avec la sélection d'une des deux laiteries. Cette dernière dispose de quatre atouts majeurs. Elle collecte déjà du lait auprès de producteurs capables d'intégrer rapidement la filière dans la mesure où leur système fourrager est déjà basé sur la distribution unique d'herbe et de fourrages secs, en particulier des agriculteurs certifiés en agriculture biologique (I6). Par ailleurs, elle se démarque par son expérience dans la fabrication de fromages au lait cru. L'entreprise en question est une PME indépendante, ce qui répond à la philosophie affichée du distributeur de participer au développement et au maintien du tissu industriel sur le territoire. Enfin, l'entreprise est déjà engagée dans des relations commerciales avec le distributeur depuis plusieurs années (I5). Pour conclure, c'est un moteur évolutionniste qui joue dans cette séquence. En effet, le distributeur poursuit sa stratégie engagée quelques années plus tôt et cherche à développer son offre de fromages sous marque filière en mettant en place un procédé classique de sélection.

\section{Séquence 2 : la mise en place de la filière (printemps 2004 - août 2005)}

Dans cette séquence, c'est un moteur téléologique qui entre en jeu : la rencontre des stratégies du distributeur et de la laiterie et le partage d'un objectif commun permettent de concrétiser le projet de création de la filière. Pour la laiterie, ce partenariat répond à l'objectif de créer un nouveau débouché commercial et de maintenir une bonne valorisation de ses produits. Elle poursuit en cela sa stratégie marketing qui repose en partie sur le développement d'une offre différenciée par la qualité. La mise en place de cette démarche de qualité est donc vue comme la suite logique des choix de production qui ont été faits auparavant en 
termes notamment de réappropriation de la production au lait cru et de valorisation de certains systèmes d'élevage.

Entre 2004 et 2005, le responsable de production de la laiterie commence à informer les exploitants, de façon individuelle, de sa volonté de créer un partenariat avec le distributeur. Il s'appuie pour cela sur les rapports privilégiés qu'il entretient de longue date avec les producteurs (I7) et développe une argumentation destinée à les inciter à intégrer la filière. Il s'adresse en premier lieu aux éleveurs qui ont conservé un système fourrager conforme aux exigences du distributeur (I6), des exploitants aux petits quotas qui avaient toujours refusé l'ensilage (entre 100000 et 150000 litres). Ainsi, en cas d'échec de construction de la filière, les producteurs ne seraient pas fortement pénalisés. Pour chaque producteur pressenti, la même procédure est appliquée : une visite préalable est réalisée par le responsable de production pour expliquer les enjeux de la filière et les principaux points du cahier des charges. Cette visite est complétée par l'audit de l'organisme tiers mandaté par le distributeur. Puis, un suivi régulier des analyses sanitaires et du maintien du respect du cahier des charges est assuré par la laiterie (face aux exigences que requiert la transformation du lait cru, les exploitations sont également sélectionnées en fonction de leurs résultats d'analyses sanitaires).

La séquence s'achève par le lancement officiel, le 6 août 2005, des premières fabrications de fromages et la signature du contrat entre la laiterie et le distributeur. On compte alors 15 producteurs, soit un volume collecté d'environ 1,5 million de litres de lait. À cette date, la coordination entre les différentes parties est assurée par le contrat qui lie le distributeur et le transformateur d'une part, et par l'engagement moral de collecte entre le transformateur et les producteurs et le versement d'une prime de $30 €$ par 1000 litres de lait tout foin d'autre part. Pour s'adapter aux contraintes du cahier des charges, la laiterie revoit l'organisation de sa collecte. Elle met en place un ramassage quotidien chez les producteurs et un système rigoureux permettant une traçabilité du lait et du fromage destinés à la filière. Des investissements sont par ailleurs réalisés au niveau des circuits de dépotage et des installations de stockage pour éviter le mélange avec d'autres types de lait. Enfin, quatre personnes supplémentaires sont recrutées.

\section{Séquence 3 : le ralentissement du développement de la filière (2006-mi 2008)}

La troisième séquence correspond à un ralentissement de la trajectoire dû au déclenchement d'un moteur dialectique. En effet, le transformateur continue à mobiliser ses relations de proximité avec les producteurs pour motiver des adhésions nouvelles (réunions, visites d'exploitation) (I7). Malgré cela, plusieurs événements participent à freiner les producteurs dans leur adhésion à la filière. Au niveau du territoire, des débats émergent au sein de la profession agricole sur l'intérêt de ce type de démarche pour plusieurs raisons. D'une part, les liens avec la grande distribution sont parfois mal perçus. Cette dernière a mauvaise presse auprès des producteurs du fait de l'âpreté des négociations commerciales (I8). Par ailleurs, l'année 2006 voit s'initier un mouvement visant à modifier le cahier des charges des AOC pour aller vers moins de contraintes sur l'alimentation des vaches (arrêt d'interdiction de l'ensilage) (I9). Dans ces conditions, la filière qualité offre moins d'attraits, étant plus restrictive pour une faible plus-value financière. D'autre part, les autres producteurs de la zone font pression sur les exploitants de la filière ou ceux qui voulaient y adhérer car cela remet parfois en cause les engagements au sein des Coopératives d'utilisation du matériel agricole (CUMA) 
d'ensilage (I10). Enfin, les systèmes fourragers excluant l'ensilage sont matière à critique et perçus par certains producteurs comme un retour en arrière (I11) (sur un territoire majoritairement passé à l'ensilage et à l'enrubannage dans les années 1990). Les craintes principales à ce sujet concernent une baisse importante de la production laitière, ne permettant plus une rentabilité suffisante des exploitations, d'autant que le système étant peu courant sur le territoire, les références et structures de conseil font défaut. L'octroi d'une aide à la modernisation des bâtiments d'élevage, incluant les outils de séchage en grange, proposée par la Région dès 2007 ne suffit pas à combattre des craintes relatives aux investissements. Des événements plus globaux viennent également influencer le cours de cette séquence. L'année 2007 est particulièrement pluvieuse, rendant difficile la récolte du foin (I12). En 2008, les producteurs sont confrontés à une forte baisse du prix du lait et les inquiétudes sont nombreuses concernant l'avenir de la filière (I13). Cette crise handicape l'ensemble des producteurs de l'entreprise de transformation, d'autant que la France entre en période de récession ce qui pénalise le pouvoir d'achat des consommateurs.

À la fin de cette séquence, le nombre de producteurs collectés dans le cadre de la filière n'a pas augmenté par rapport à l'effectif au lancement de la filière.

\section{Séquence 4 : une bifurcation dans la trajectoire (mi 2008-2010)}

En 2008, la trajectoire de la démarche connaît une bifurcation liée à l'accroissement notable des débouchés potentiels pour les produits sous marque filière. Le distributeur uniformise les enseignes de l'ensemble de ses magasins, augmentant ainsi le nombre de supermarchés ayant des rayons à la coupe et pouvant commercialiser des produits sous ses marques propres (I14). Cette évolution a pour conséquence directe une augmentation du potentiel de volume de fromages commercialisés. Par ailleurs, les produits de la filière sont également référencés sous une autre marque propre du distributeur, possédant une plus forte renommée auprès des consommateurs. Ce contexte favorable conduit à l'enclenchement d'un moteur téléologique avec à nouveau une convergence d'intérêt entre les différentes parties prenantes.

Cette séquence repose sur une succession d'actions conjointes menées par le distributeur et la laiterie. Ils mettent en place une plus forte coordination entre eux pour développer une stratégie incitative auprès des producteurs. Ils réalisent ensemble des visites dans les exploitations candidates pour faire prendre conscience aux producteurs des avantages de l'appartenance à la filière. Des producteurs déjà adhérents à la filière sont invités au Salon international de l'agriculture à Paris pour faire la promotion de leurs produits et des réunions sont organisées entre les producteurs adhérents et les candidats potentiels. Ces réunions constituent de véritables lieux d'échanges sur les avantages de l'adhésion à la filière. Le responsable de production s'appuie sur des exemples de systèmes tout foin performants pour séduire de nouveaux éleveurs (I15). Il organise des visites dans des exploitations converties au système tout foin sans pour autant avoir de prime supplémentaire pour l'achat de leur lait. Pendant la visite, leur rentabilité économique est mise en avant. Par la suite, une véritable analyse prévisionnelle de la rentabilité économique des exploitations candidates est réalisée pour appuyer l'argumentaire. Ces échanges renforcent la confiance entre le distributeur et les producteurs. De plus, les relations et les échanges entre producteurs se multiplient, entraînant la diffusion de la technique du séchage en grange, qui se concrétise par la création un réseau d'échanges sur cette technique. Ces évolutions permettent de 
lever les dernières réticences des producteurs qui craignaient d'être confrontés à des difficultés liées à la récolte de foin et à une baisse de la productivité liée à des fourrages de qualité inférieure à ceux fournis par l'ensilage.

Cette séquence aboutit à des contrats formalisés par la laiterie assurant aux producteurs une prime de 60 euros par 1000 litres de lait et la collecte de leur lait pour une durée de 7 ans qui permet de garantir le retour sur investissement en cas de conversion au séchage en grange. En l'espace d'à peine 3 ans, 11 exploitants adhèrent à la filière, dont 5 possèdent des références laitières supérieures à 250000 litres de lait. Ceci permet au transformateur d'asseoir la filière et de s'adresser désormais à des exploitants qui présentent des perspectives d'évolution compatibles avec le maintien des volumes transformés à long terme. Dès lors, des producteurs à l'exploitation de taille plus importante souhaitent concilier agrandissement et maintien de la rentabilité économique de leur structure à long terme, avec une évolution de la main-d'œuvre et s'investissent dans la filière.

\section{Séquence 5 : vers un nouveau ralentissement de la filière (2011-2012)}

Dans cette dernière séquence, les stratégies des producteurs et de la laiterie s'opposent pour expliquer un nouveau ralentissement traduisant l'existence d'un moteur dialectique.

Trois éléments participent à rendre la filière attractive pour les éleveurs. Les réseaux d'échange sur la technique du séchage en grange qui participent au renforcement des relations entre producteurs et à la diffusion des atouts de la filière (I16). Par ailleurs, le contexte territorial est favorable à une évolution vers des prairies naturelles (I17) : dans les AOP auvergnates, des volontés s'expriment pour faire évoluer les cahiers des charges vers une réduction de l'utilisation d'ensilage et des outils de gestion des prairies naturelles sont proposés. En parallèle, les organismes de conseil collaborent pour proposer aux producteurs de nouvelles références sur les systèmes tout foin. Ils contribuent fortement à développer la volonté de certains producteurs de diversifier leur revenu afin de concilier agrandissement de leur structure et évolution de la main-d'œuvre. Plus globalement, la perspective de suppression des quotas laitiers en 2015 (I18) joue sur la volonté d'engagement des producteurs dans la filière - la filière qualité étant vue par les producteurs -, mais également par les acteurs du développement territorial, comme un moyen de sécuriser leurs débouchés.

Cependant, le renforcement de cette attractivité s'oppose à la nouvelle stratégie de la laiterie qui souhaite désormais gérer ses volumes de façon à éviter les excédents pour éviter de pénaliser son revenu ou celui des producteurs. En 2010 en effet, la laiterie collecte quasiment les litrages suffisants pour satisfaire la demande du distributeur. À cela s'ajoutent les difficultés que connaît le distributeur à cette période (perte de parts de marché) et les évolutions de sa stratégie (le développement de filières qualité ne semble plus prioritaire) (I19).

En conséquence et malgré la motivation des producteurs, la laiterie refuse l'adhésion de nouveaux producteurs. En 2012, ils sont au nombre de 19 et la quantité annuelle de lait collecté stagne à 300000 litres.

\section{Discussion}

L'analyse des ingrédients et des moteurs qui interviennent dans les séquences permet de saisir la façon dont se construit la relation entre le distributeur, la laiterie et les éleveurs et les variables qui influencent le processus. Elle permet également de mettre en évidence les interactions entre les différents éléments qui composent la relation. 
Cette analyse souligne l'influence que peuvent avoir les différentes parties prenantes dans la trajectoire de la filière qualité. On confirme bien le principe selon lequel toutes les parties sont actives dans la construction de la relation (Skytte, 2010). En effet, même si le distributeur peut être considéré comme l'initiateur de la démarche, on observe qu'au cours des différentes séquences, la laiterie (séquence 2 et 5 notamment) ainsi que les éleveurs (séquence 3 et 4 ) jouent sur le rythme de développement de la démarche. Distributeurs et fournisseurs agissent sur la trajectoire de la filière, avec des rôles différents, variables au fil du temps. Néanmoins, on peut noter le rôle pivot de la laiterie à la lumière des dispositifs qu'elle met en place pour assurer le maintien de la relation qui renvoie à la notion d'engagement développée notamment par Morgan et Hunt (1994). En effet, au départ de la relation, elle réalise un certain nombre d'investissements (organisation de la collecte du lait, outil de transformation, embauches, etc.) pour pouvoir répondre au cahier des charges de la filière. Par ailleurs, elle met en place un dispositif de recrutement de nouveaux éleveurs pour assurer le développement suffisant de la filière pour répondre aux besoins du distributeur. Enfin, cet engagement se traduit également par une formalisation des échanges avec ses fournisseurs à travers la mise en place de contrats qui garantissent la collecte et le versement d'une prime pour les éleveurs sur une durée correspondant à celle de l'amortissement des investissements pour le matériel de séchage en grange.

L'analyse de la trajectoire de la filière montre que le rôle des acteurs dans l'évolution de la relation est lié non seulement à leurs caractéristiques propres, et notamment à leurs stratégies, mais également à l'atmosphère de la relation. Ainsi, la convergence des intérêts des parties et le climat de confiance existant entre elles (mauvaise opinion des producteurs sur le distributeur, importance de l'ancienneté des relations éleveurs-laiterie, etc.) apparaissent centraux. De la même façon, des conflits (au sens d'une situation dans laquelle un membre du canal considère qu'un autre membre a adopté un comportement qui l'empêche d'atteindre ses propres objectifs) (Filser, des Garets et Paché, 2001) participent de la trajectoire de la démarche (divergence de perception sur la technique du séchage en grange en séquence 3 , incompatibilité d'objectifs en séquence 5).

L'analyse processuelle montre également l'importance de la communication, non seulement entre les producteurs, la laiterie et le distributeur, mais également le poids de l'existence d'échanges informationnels et sociaux au sein d'un même groupe d'acteurs, en l'occurrence les éleveurs (réseau d'échange sur le tout foin). Ce dernier élément souligne également le fait que la relation s'inscrit dans un ensemble de relations qui entrent en interaction (Ford et Håkansson, 2006)

$$
\begin{gathered}
* \\
* \quad *
\end{gathered}
$$

L'analyse processuelle appliquée à un cas de filière qualité éclaire la façon dont se construit une relation de coopération entre un distributeur et ses fournisseurs. Plus précisément, ce travail permet de souligner le rôle des différents acteurs dans la construction de la relation, les différentes phases dans la construction d'une relation de coopération entre un distributeur et ses fournisseurs et la façon dont conflit et coopération s'articulent et jouent sur cette trajectoire, ainsi que les éléments de l'environnement de la relation qui contribuent à la construction de la démarche. Ainsi, l'analyse confirme le fait que les interactions entre acteurs se placent dans une histoire et sont liées notamment aux interactions passées. 
En conclusion, sans remettre en cause le déséquilibre des rapports de force avec les distributeurs dans les filières agroalimentaires, ce travail nuance l'idée d'une approche uniquement conflictuelle des relations fournisseurs-distributeurs, l'évolution du contexte concurrentiel dans lequel se trouve la grande distribution l'obligeant à construire des relations de long terme avec ses fournisseurs et à développer des approches plus relationnelles.

Dans le cadre des travaux sur les relations producteurs-distributeurs agroalimentaires, ce travail permet ainsi de mieux comprendre l'articulation des éléments de contexte, d'atmosphère, de caractéristiques et de comportement des acteurs dans la mise en place et le développement d'une relation de long terme.

Plus largement, cette recherche permet d'éclairer la question des interactions entre la grande distribution et les acteurs de la production agricole et agroalimentaire. Elle soulève notamment la question du rôle des distributeurs dans la construction territoriale à travers leur implication dans les systèmes agroalimentaires locaux. L'analyse de la trajectoire laisse transparaître à plusieurs reprises la façon dont le distributeur mobilise des ressources locales pour mettre en œuvre sa stratégie (AOP, savoir-faire et relations sociales). Une analyse à une plus large échelle permettrait de mieux saisir la nature des ressources locales mobilisées ainsi que la façon dont ces stratégies agissent sur les dynamiques territoriales (structuration des systèmes alimentaires, retombées économiques).

Les résultats présentés dans cet article sont issus d'une recherche conduite dans le cadre du projet ANR 2010 STRA 005 MOUVE.

Les auteures remercient Amélie Fercoq pour sa contribution à cette recherche ainsi que les relecteurs anonymes pour leurs commentaires enrichissants qui ont permis de finaliser cet article.

\section{RÉFÉRENCES BIBLIOGRAPHIQUES}

Allain M.-L., Chambolle C. (2003). Les relations entre la grande distribution et ses fournisseurs : bilan et limites de trente ans de régulation. Revue française d'économie, p. $169-212$.

Antéblian-Lambrey B. (1998). L'évolution des modèles d'analyse en marketing de la distribution et la prise en compte de nouveaux concepts dans la pratique stratégique des firmes. Revue Française de Marketing, vol. 5, n 170, p. 27-39.

Berges-Sennou F., Caprice S. (2003). Les rapports producteurs-distributeurs : fondements et implications de la puissance d'achat. Économie rurale, $\mathrm{n}^{\circ}$ 277-278, p. 192-205.

Beylier R.-P., Messeghem K., Fort F. (2012), « Rôle des MDD de terroir dans la construction de la légitimité des distributeurs. Decisions Marketing, $\mathrm{n}^{\circ}$ 66, p. 35-45.
Bidart C., Brochier D., Correia M., Garnier J., Gilson A., Longo M.E., Mendez A., Mercier D., Oiry E., Pascal A., Pérocheau G., Tchobanian R. (2010). Le système et la méthode. In Mendez A. (dir.), Processus. Concepts et méthode pour l'analyse temporelle en sciences sociales, Louvain-laNeuve, Academia Bruylant, p. 219-242.

Bizzi L., Langley A. (2012). Studying processes in and around networks. Industrial Marketing Management, vol. 41, $\mathrm{n}^{\circ} 2$, p. 224-234.

Brochier D., Garnier J., Gilson A., Longo M.E., Mendez A., Mercier D., Pascal A., Perocheau G., Tchobanian R., Oiry E., Bidart C. (2010). Propositions pour un cadre théorique unifié et une méthodologie d'analyse des trajectoires des projets dans les organisations. Management \& Avenir, vol. 36, $\mathrm{n}^{\circ} 6$, p. $84-84$. 
Burch D., Lawrence G. (2005). Supermarket own brands, supply chains and the transformation of the agri-food system. International Journal of Sociology of Agriculture and Food, vol. 13, n 1 , p. 1-18.

Burch D., Lawrence G. (dir.) (2007). Supermarkets in agri-food supply chains: transformations in the production and consumption of foods, Cheltenham UK, Northampton MA, Edward Elgar Publishing, $330 \mathrm{p}$.

Cova B., Salle R. (1992). L'évolution de la modélisation du comportement d'achat industriel : panorama des nouveaux courants de recherche. Recherche et Applications en Marketing, vol. VII, $\mathrm{n}^{\circ}$ 2/92, p. 83-106.

Cox A., Chicksand D. (2007). Are win-wins feasible? Power relationships in agri-food supply chains and markets. In Burch D., Lawrence G. (dir.), Supermarkets in agrifood supply chains: transformations in the production and consumption of foods, Cheltenham UK, Northampton MA, Edward Elgar Publishing, p. 74-99.

Daumas J.C. (2006). Consommation de masse et grande distribution : une révolution permanente (1957-2005). Vingtième siècle. Revue d'histoire, $\mathrm{n}^{\circ}$ 91, p. 57-76.

Dwyer F. R., Schurr P. H., Oh S. (1987). Developing Buyer-Seller Relationships. Journal of Marketing, vol. 51, $\mathrm{n}^{\circ}$ 2, p. 11-27.

Filser M. (2000). Les théories du canal de distribution : le dualisme des paradigmes. In Fabbe- Costes N., Colin J., Paché G. (dir.), Faire de la recherche en logistique et distribution?, Paris, Vuibert, p. 55-89.

Filser M., Garets V. des, Paché G. (2001), La distribution : organisation et stratégie. Paris, EMS.

Filser M., Paché G. (2008). La dynamique des canaux de distribution. Revue française de gestion, vol. 182, n 2, p. 109-133.

Fonte M. (2008). Knowledge, Food and Place. A Way of Producing, a Way of Knowing. Sociologia Ruralis, vol. 48, n 3, p. 200-222.

Fontguyon G. de, Giraud-Héraud É., Rouached L., Soler L.-G. (2003). Qualité des produits alimentaires et marques de filières. Sociologie du Travail, vol. 45, $\mathrm{n}^{\circ} 1$, p. 77-94.
Ford D., Håkansson H. (2006). The idea of interaction. The IMP Journal, vol. 1, $\mathrm{n}^{\circ} 1$, p. 4-20.

Håkansson H. (1982). International marketing and purchasing of industrial goods: an interaction approach. Chichester, New York, Wiley.

Halinen A., Medlin C. J., Törnroos J.-Å. (2012). Time and process in business network research. Industrial Marketing Management, vol. $41, \mathrm{n}^{\circ} 2$, p. $215-223$.

Hunt S. D., Ray N. M., Wood R. Van (1985). Behavioral dimensions of channels of distribution: review and synthesis. Journal of the Academy of Marketing Science, vol. 13, $\mathrm{n}^{\circ} 3$, p. 1-24.

Leek S., Turnbull P. W., Naudé P. (2001). Interactions, relationships and networks-past, present and future. The 17th International Conference on Industrial Marketing and Purchasing. Oslo, Norway.

Lepers X. (2003). Vers une nouvelle conceptualisation de la relation d'échange fournisseurs-grands distributeurs. Revue Française de Gestion, vol. 29, n ${ }^{\circ}$ 143, p. 81-94.

Longo M.-E., Mendez A., Tchobanian R. (2010). Le découpage temporel du processus : l'analyse par séquences. In Mendez A. (dir.), Processus. Concepts et méthode pour l'analyse temporelle en sciences sociales, Louvainla-Neuve, Academia Bruylant, p. 73-89.

Mazé A. (2002). Retailers' branding strategies: Contract design, organisational change and learning. Journal on Chain and Network Science, vol. 2, $\mathrm{n}^{\circ}$ 1, p. 33-45.

Medlin C. J. (2004). Interaction in business relationships: A time perspective. Industrial Marketing Management, vol. 33, $\mathrm{n}^{\circ} 3$, p. 185-193.

Mendez A. (dir.) (2010). Processus. Concepts et méthode pour l'analyse temporelle en sciences sociales. Louvain-la-Neuve, Academia Bruylant.

Mercier D., Oiry E. (2010). Le contexte et ses ingrédients dans l'analyse de processus : conceptualisation et méthode. In Mendez A. (dir.), Processus. Concepts et méthode pour l'analyse temporelle en sciences sociales, Louvain-la-Neuve, Academia Bruylant, p. 29-42. 
Messeghem K. (2005). Les distributeurs en quête de légitimité : le cas des accords de coopération avec les PME. Retailers looking for legitimacy: The case of cooperation agreements with SMEs, $\mathrm{n}^{\circ} 39$, p. 57-66.

Morgan R. M., Hunt S. D. (1994). The Commitment-Trust Theory of Relationship Marketing. Journal of Marketing, vol. 58, $\mathrm{n}^{\circ} 3$, p. 20.

Nogatchewsky G., Donada C. (2005), « Vingt ans de recherches empiriques en marketing sur la performance des relations clientfournisseur. Recherche et Application en Marketing, vol. 20, n 20, 4, p. 71-96.

Pérocheau G., Correia M. (2010). Les moteurs, principes génératifs du mouvement dans les processus. In Mendez A. (dir.), Processus. Concepts et méthode pour l'analyse temporelle en sciences sociales, Louvain-la-Neuve, Academia Bruylant, p. 123-139.

Pettigrew A. M. (1997). What is a processual analysis? Scandinavian Journal of Management, vol. 13, $\mathrm{n}^{\circ}$ 4, p. 337-348.

Pouzenc M., Guibert N. (2010). Produits et filières : entre le rentable et l'équitable. In Dugot P., Pouzenc M. (dir.), Territoires $d u$ commerce et développement durable, Paris, L'Harmattan, p. 191-236.
Réviron S., Chappuis J.-M. (2005). Effects of the Swiss retailers' strategy on the governance structure of the fresh food products supply chains, Agribusiness, vol. 21, $\mathrm{n}^{\circ} 2$, p. 237-252.

Ropo A., Eriksson P., Hunt J. G. (1997). Reflections on conducting processual research on management and organizations. Scandinavian Journal of Management, vol. $13, n^{\circ} 4$, p. 331-335.

Sabri R., Messeghem K. (2012). Accompagnement des petits producteurs par la grande distribution : le cas de "Marjane Holding ». Marché et organisations, vol. 15, $\mathrm{n}^{\circ}$ 1, p. 143-168.

Skytte H. (2010). Relationships between food producers and retailers. 26th Industrial Marketing and Purchasing Group Conference, Budapest.

Stern L. W. (dir.) (1969). Distribution channels: behavioral dimensions. Boston, Houghton Mifflin Company.

Van de Ven A. H., Poole M.S. (1995). Explaining Development and Change in Organizations. Academy of Management Review, vol. 20, n 3 , p. 510-540.

Wilkinson I. (2001). A History of Network and Channels Thinking in Marketing in the 20th Century. Australasian Marketing Journal, vol. 9, $\mathrm{n}^{\circ}$ 2, p. 23-52. 